\title{
Making a choice when information is missing: The case of medical versus consumer choices
}

\author{
Cristina Maroiu ${ }^{1}$ \& Laurențiu P. Maricuțoiu ${ }^{1 *}$ \\ ${ }^{1}$ Department of Psychology, West University of Timişoara, Romania.
}

Received 11.04.2019; Received revised 7.06.2019; Accepted 15.06.2019

Available online 30.06.2019

\begin{abstract}
This paper reports an experimental investigation of decision-making under uncertainty. Today, patients are encouraged to participate, or even decide for themselves what kind of care and treatment they should get; they are now in the position of a consumer that chooses what he believes is best. However, choosing a specific treatment rather than another has more important consequences in one's life than choosing a brand of yogurt rather than another. We wanted to see if people choose according to the available information, or not. Using a within-person design, we asked if there are any differences in the way people make medical choices, as compared to nonmedical, neutral choices. Latency was also measured for the 21 choices each participant had to make. Additionally, we investigated moral purity, and gender - as between-person variables, to see if they play a role in decision-making under uncertainty. We analyzed the data with hierarchical linear modelling, where a series of choices (level 1) were nested within individuals (level 2). Results showed that people relied on the available information more often for medical choices than for non-medical, neutral choices. The less time spent, the higher the probability of relying on the available information. Also, while moral purity was not a significant predictor of decision-making, gender seems to be a moderator: men tend to rely more than women on the known information, in the case of medical choices, while in the case of non-medical choices, the differences between men and women are insignificant.
\end{abstract}

Keywords: decision making, uncertainty, medical treatments, consumer choice, patient choice.

Address of correspondence: Laurențiu P. Maricuțoiu, Department of Psychology, West University of Timișoara, 4 Vasile Pârvan Blvd., Room 303, 300223 Timișoara, Romania. E-mail: laurentiu.maricutoiu@e-uvt.ro

\section{Introduction}

Every day, we have to make decisions. We have to decide if we should ride our bike or drive to work, if we should have dessert or not, watch a movie or read a book instead, and so on. Sometimes, our decisions have significant consequences on our lives, like when we have to make a career choice, buy a house, or decide which treatment we should take if we have a medical issue. According to Gigerenzer, (2008), we live in an uncertain world, and there are many situations where we need to make decisions based on limited information.

Most daily choices involve some degree of missing information (Kivetz \& Simonson, 2000), and Figure 1 shows a mere example of these situations. The scenario in Figure 1 presents us with two options. These options have common (i.e., client ratings) and unique attributes (i.e., distance and price range). So far, research found that people tend to give more weight to the common attributes as compared to unique attributes (Slovic and MacPhillamy, 1974). According to Kivetz \& Simonson (2000), this is because common attributes enable the direct comparison of two options on the same scale (e.g., which option has better client ratings). To include the unique attributes in the comparison would involve an evaluation of the attribute's utility, as well as formulating inferences about missing values (Kivetz \& Simonson, 2000) - in other words, it would require more effort.

Decision-making with incomplete information was mainly investigated in the domain of consumer choice (Kivetz \& Simonson, 2000), even though the medical field also presents similar situations. Frosch \& Kaplan (1999) argued that many, if not most of the medical treatments have a somewhat degree of uncertainty regarding 
outcomes. In their work, physicians have to rely on incomplete research bases, sometimes affected by selection and reporting bias (Chalmers \& Glaziou, 2009). Moreover, the medical decision is not limited to the physicians' decision, because contemporary medicine advocates that the patient should play an active role in the decisionmaking process regarding their care (Charles, Ghafni, \&Whelan, 1997).

Assuming you want to go to a restaurant to have lunch. Please analyze the following options and choose the one you consider best.

\begin{tabular}{rcc}
\cline { 2 - 3 } & Restaurant A & Restaurant $\mathbf{B}$ \\
\cline { 2 - 3 } Client ratings & 5 out of 5 & 3 out of 5 \\
Distance & Unavailable information & 15 minute drive \\
Price range & Medium prices & Unavailable information \\
\cline { 2 - 3 } & Figure 1. Stimuli for the decision task- non-medical venue &
\end{tabular}

Figure 1. Stimuli for the decision task- non-medical venue

According to Politi, Han, \& Nananda (2007), research has yet to provide us with best practices when it comes to communicating uncertainty to patients regarding the harms and benefits of possible treatments. Frosch \& Kaplan (1999) suggested that further research is necessary in order to clarify how people think about the possible treatments they can choose. Therefore, in order to recommend best practices for dealing with uncertainty, we need to have a better understanding of the patients' decisional process.

In this paper, we aim to enhance our understanding of how people make choices under uncertainty. While we do have some idea on how people make consumer choices under uncertainty (Kivetz \& Simonson, 2000), medical choices represent a different situation because of their consequences on people's lives, beyond material gains or losses. Thus, we want to verify if people approach medical choices under uncertainty in the same way they approach consumer choices under uncertainty. Also, we want to investigate latency and the endorsement of moral purity as possible predictors of choices under uncertainty.

\section{Medical vs. non-medical decision}

Previous research suggested that people's strategies for coping with uncertainty vary according to the situations they are facing (Lipshitz \& Strauss, 1997). Current theories of decision-making (e.g., prospect theory) show that instead of referring to the objective outcome, people base their decisions on the potential value of losses and gains (Tversky \& Kahneman, 1992). During the decisional process, the person has expectations about feelings they might experience later (Connolly \& Zeelenberg, 2002), so it is possible that anticipated regret might shift one's choice strategy. The different decisions one makes vary in terms of their potential impact, and medical decisions have high stakes for one's future health and wellbeing. For instance, while choosing the wrong brand of a consumer product probably has a small impact in one's life, choosing what to do when a screening test reveals signs of cancer could mean a life-or-death situation (Kahn \& Luce, 2003). Because medical decisions have essential consequences in one's life, we believe that individuals will tend to acknowledge uncertainty and rely on the known information (common attributes of the alternatives). Hence, we formulate the following hypothesis:

H1. People tend to choose the option that is superior on the common attribute more often when making medical choices, as compared to non-medical choices.

Response time
When it comes to decision-making, another important variable is the time one takes to make a decision. Response time (latency) can help us understand the inner processes of one's judgment, and this idea is as old as psychology itself (Heitz, 2014). The speed-accuracy tradeoff (Keramati, Dezfouli \& Piray, 2011) states that usually, the less time we spend deliberating on a decision, the higher the probability of a bad outcome. However, some researchers (Gigerenzer \& Todd, 1999) claim that there are many situations where fast and frugal strategies lead to better outcomes. While the question of what is a good decision is beyond the scope of this paper, we do expect to find a relationship between the decision outcome and the time needed to take that particular decision. Previous research showed that using unique attributes when comparing options is a more demanding process, as compared with relying on common attributes to make our choice (Kivetz \& Simonson, 2000). Since demanding tasks usually require more time to solve, it is possible to find that larger decision time lags are associated with decisions based on the unique attributes, while shorter decision time lags predict decisions based on common attributes. Consequently, we expect that:

H2. Response latency predicts people's tendency to choose the option that is superior on the common attribute.

\section{Moral orientation}

Recent research focused on the idea that moral values play a role in people's attitudes and judgments. Rutjens, Sutton, \& van der Lee (2018) propose that "Moral judgments might interfere with factual interpretations of the scientific evidence presented, leading to increased skepticism and rejection." The moral foundation purity seems to play a part in people's decision making when it comes to medical treatments. For instance, Amin et al. (2017) showed that moral purity is significantly associated with vaccine hesitancy. Furthermore, out of all the moral foundations (Harm/care, Fairness/reciprocity, Ingroup/loyalty, Authority/respect, and Purity/sanctity), Purity was the only foundation that predicted science support (Rutjens et al., 2018). People that value purity are preoccupied with avoiding anything that is disgusting or unnatural; in this vein, one possible way of thinking is that nature does things well, thus we should not interfere (Dubé et al., 2016). Medical treatments are interventions aimed at improving the patient's health so, in a certain way, they might be regarded as an interference with nature. Because research suggests that the endorsement of purity is related to the way people judge information regarding medical 
treatments (Amin et al. 2017; Rutjens et al.,2018), we expect that:

H3: The tendency to endorse purity as moral value is linked to the way people process common vs. unique attributes.

\section{Gender differences}

Research shows that when it comes to decisionmaking, men and women do not think alike. For instance, Say, Murtagh \& Thomson (2006) showed that women were more likely than men to prefer an active role in decisions about their medical treatment. Regarding non-medical decisions, Croson \& Gneezy (2009) reported gender differences regarding risk aversion (i.e., women are more risk-averse), social preferences (e.g., women are more sensitive to social cues when it comes to determining appropriate behavior), and competitiveness (i.e., women are less prone to enter competitive situations). Since previous research found differences between men and women, both in medical and in non-medical decisionmaking, we hypothesize that:

H4: Gender is a significant predictor of people's tendency to base their decision on common attributes, both for medical and non-medical decisions.

\section{Method}

\section{Participants}

First-year students enrolled at the Faculty of Psychology within the West University of Timisoara received via e-mail an invitation to participate in this research. They were informed that the research would last between 20 and 30 minutes, and they will have to fill in a series of questionnaires, and a decision task. The final sample was composed of 65 participants (55\% males), $\mathrm{M}_{\text {age }}=20.2$ years $\mathrm{SD}=1.1$. The students were rewarded with course credit for their participation.

\section{Design}

We employed a mixed design, with both within- (L1) and between- subjects variables (L2). The within-subject variables are decision type (medical vs. non-medical), and latency- this is a repeated-measure variable, as we measured latency for each of the 21 decisions the participants had to make. The between-subjects predictors are the moral value purity and gender. The dependent variable is the decision outcome (decision-based on the common vs. unique attributes).

\section{Measures}

The decision task assessed whether the participants tend to rely either on the common attributes or on the unique attributes when making a choice. Each choice holds two known attribute information and one unknown attribute information. When comparing any two options, the participants will have one common attribute (e.g., for medical-related choice 1 and choice 2, the disadvantages of the treatments are known), and two unique attributes (e.g., for treatment 1 , the information about advantages are known, and for treatment 2 unknown, while at the same time the information about costs is known for treatment 1 , and unknown for treatment 2). See Figure 2 for an example of a medical scenario, and Figure 1 for an example of a non-medical scenario. Deciding based on the common attributes means to compare the information that is known for both options (e.g., which option has the smaller disadvantage), while deciding based on the unique attributes means to compare the information that is known for one option, but unknown for the other (e.g., which option has the bigger advantage).

Response latencies represent the time it took until participants chose each of the 21 decision trials. Thus, we had 21 response latencies for each participant. The decision task was delivered though Inquisit software, and there was no time limit to solving it. Trial number was considered in order to control for the possibility of task habituation. Because each of the 21 pairs of consecutive choices had the same structure, it was possible that their answers would be influenced by the trial number rather than the independent variables (e.g., it was possible that response latency would decrease as trials succeed). In order to statistically control this possibility, we considered the trial number of each choice the participants made. Moral Foundations Questionnaire (Graham et al., 2011) was used to assess individual differences regarding Purity concerns. There are six items that measure Purity, and the answers are on a scale from 0 (Not at all relevant/ Strongly disagree/) to 5 (Extremely relevant/ Strongly agree). The internal consistency for the Purity dimension was acceptable (Cronbach's alpha $=.69)$

Assuming that within your community some peers have a severe type of flu. Thus, it is possible that you might get it also, and complications can be deadly. Please analyze the following options and choose the one you would endorse.

\begin{tabular}{|c|c|c|}
\hline & The choice to vaccinate & Take homeopathic preventive measures \\
\hline Advantage & $98 \%$ chances of avoiding any clinical symptoms & Unavailable information \\
\hline Disadvantage & $\begin{array}{l}0.1 \% \text { chances of side effects (e.g., muscle pain, } \\
\text { fever) }\end{array}$ & $8 \%$ chances of side effects (e.g., insomnia, nausea) \\
\hline Costs & Unavailable information & High costs \\
\hline
\end{tabular}

\section{Procedure and manipulations}

The participants completed self-report measures for moral foundations, gender and age, followed by a decision task: a series of medical- related choices and a series of non-medical-related choices (described below). We collected data in the laboratory, with the help of the
Inquisit software; the completion of all measures took between 20-30 minutes.

Type of decision was manipulated through the content of the decision task: half of the decisions referred to medical scenarios, and the other half referred to nonmedical, everyday situations. We had a total of eight hypothetical situations, four medical related and four non- 
medical-related; all are presented in Appendix A. For each situation, we defined three options, and the participants had to compare the options in pairs (option 1 vs. option 2, option 1 vs. option 3 , option 2 vs. option 3), and choose the one they would adhere to. This amounts to a total of 3 (choices for each situation) $* 4=12$ medical related choices, and 12 non-medical related choices. Because one of the medical situations had little variability in responses, we excluded it and remained with three medical situations ( 9 choices) and four non-medical situations (12 choices) 21 choices in total.

\section{Data analysis}

We used hierarchical linear modeling with a dichotomic outcome (Bernoulli distribution) to analyze a data structure where the 21 decisions each subject had to make (level 1) were nested within subjects (level 2). The analysis was performed with the HLM software. The within-subject predictors (i.e., Level 1) were: decision type (medical or non-medical) - corresponding to H1, response latency-corresponding to $\mathrm{H} 2$, and trial number (this was to control for the possibility of task habituation). In order to ease the interpretation of the results, response latency and trial number were centered around the participants' mean. The between-subject predictors (i.e., Level 2) were the endorsement of purity as moral value (corresponding to $\mathrm{H} 3$ ), and gender (corresponding to H4). The betweensubjects predictors were centered around the grand mean.

Model testing proceeded in 4 phases: the unconstrained (null) model, the fixed effects model, the random slopes model, means- as outcome model, and intercepts and slopes as outcome model.

\section{Results}

Initial analyses indicated that our participants' decisions were primarily based on known information. On average, $75.65 \%$ of the decision taken by our participants were based on known information, which suggests that the odds of deciding based on known information were more than double as compared with the odds of deciding on unknown information (Odds ratio $=2.13$ ). The individual decisions based on known information ranged between $38.10 \%$ and $85.71 \%$. However, the estimation of the variance components indicated that the interpersonal differences are not statistically significant $\left(\chi^{2}(64)=73.17, p=.20\right)$, which suggests that it is unlikely to find interpersonal predictors (i.e., the level 2 predictors).

\section{The within-subject predictors}

The random- regression coefficients model was tested using the variables: Trial number, Latency, and Type of decision (medical or non-medical). Table 1 shows absolute effects, for each predictor individually;

The type of decision had a significant effect $\left(\gamma_{10}=-.40\right.$, $p<.05$ ) on the information used by our participants to formulate their decision, and this result supported the H1. The negative effect indicated that our participants relied more on the common attributes when faced with medical decisions than in the case of non-medical decisions. Thus, for medical decisions, there was a $73.12 \%$ chance that people would make their choice based on the known information, while for the non-medical decision, there was a $65.57 \%$ chance that people would make their choice based on the known information. We calculated these probabilities based on the algorithm presented by (Rousell \& Emenogu, 2009, pp. 1-9).

Table 1. Results from the random intercepts model: individual effect of each predictor

\begin{tabular}{cccc}
\hline Fixed effect & $\begin{array}{c}\text { Coefficient } \\
(\text { S.E) }\end{array}$ & $\begin{array}{c}\text { t-ratio } \\
\text { (d.f.) }\end{array}$ & $\begin{array}{c}\text { p- } \\
\text { value }\end{array}$ \\
\hline Trial number $\left(\gamma_{10}\right)$ & $0.03(0.02)$ & $2.19(64)$ & .03 \\
Response latency- $\left(\gamma_{20}\right)$ & -0.000013 & $-2.6(1295)$ & .009 \\
Decision type $\left(\gamma_{30}\right)$ & $-0.000005)$ & $-1.75(64)$ & .084 \\
\hline
\end{tabular}

Response latency $\left(\gamma_{20}=-.000018, \mathrm{p}<.001\right)$ had a significant effect, in the sense that the fewer time people took to deliberate, the more they tended to rely on the common attributes when making a choice. This result supported Hypothesis 2.

Trial number $\left(\gamma_{10}=.01, p=.36\right)$ had no relative effect on the type of decision people made, so we can say there was no learning effect in this case.

\section{The between-subjects predictors}

We used the between-subjects (or Level 2) predictors i) to explain the participants' tendency to rely on known information (i.e., we predicted the intercepts); and ii) to explain any significant variation of the slopes estimated for the intra-personal predictors. We tested the following L2 predictor variables: Purity and Gender. These betweensubjects variables did not predict the participants' general tendency to rely on known information: $\gamma_{01}=-0.02, \mathrm{SE}=$ $.01, \mathrm{p}=.11$ (for Purity), and $\gamma_{02}=-0.12, \mathrm{SE}=.12, \mathrm{p}=.33$ (for Gender); See Table 2.

Table 2. Individual (zero-order) effect of each Level 2 predictor

\begin{tabular}{cccc}
\hline Fixed effect & Coefficient (S.E) & t-ratio (d.f.) & p-value \\
\hline Gender $\left(\gamma_{01}\right)$ & $-0.12(0.12)$ & $-0.97(63)$ & .33 \\
Purity $\left(\gamma_{20}\right)$ & $-0.02(0.01)$ & $-1.59(63)$ & .12 \\
\hline
\end{tabular}

Regarding the prediction of the intra-personal slopes, we found that Gender $\left(\gamma_{31}=0.56\right.$, SE $\left.=.27 \mathrm{p}<.001\right)$ moderates the relationship between the type of decision (i.e., medical vs. non-medical) and using common attributes when formulating a decision.

This result suggests that men tend to rely more than women on the common attribute, in the case of medical choices, while in the case of non-medical choices, the differences between men and women are insignificant. Specifically, when faced with a medical decision, there is a $76.63 \%$ chance that men will rely on the known information when making a choice, while for women there is a smaller chance that they will do so: $69.23 \%$. For the non-medical choices, there is no statistically significant difference between men and women. See Table 3 for coefficients.

The final equation is:

Decision $=\gamma_{00}+\gamma_{10}$ *Type of decision $+\gamma_{11}$ *gender* Type of decision $+\gamma_{20} *$ latency $+\mathrm{u}_{0 \mathrm{j}}+\mathrm{u}_{1 \mathrm{j}} *$ Type of decision $+\mathrm{r}_{\mathrm{ij}}$ 
Table 3. Results from the intercepts and slopes as outcomes model

\begin{tabular}{|c|c|c|c|}
\hline Fixed effect & Coefficient (S.E) & t-ratio $(d . f)$. & p-value \\
\hline \multicolumn{4}{|l|}{ Level 1} \\
\hline Intercept, $\gamma_{00}$ & $0.65(0.14)$ & $4.59(64)$ & $<.001$ \\
\hline Trial number $\gamma_{10}$ & $0.01(0.01)$ & $0.85(64)$ & .4 \\
\hline Latency $\gamma_{20}$ & $-0.000018(0.000005)$ & $-3.48(1165)$ & $<.001$ \\
\hline Type of decision $\gamma_{30}$ & $-1.22(0.36)$ & $-3.39(63)$ & .001 \\
\hline Gender $\gamma_{31}$ & $0.56(0.27)$ & $2.01(63)$ & .04 \\
\hline & Random parameters & & \\
\hline & Variance & & $\mathrm{p}$-value \\
\hline Level 1 residual variance $-\operatorname{Var}\left(\mathrm{u}_{0 \mathrm{j}}\right)$ & $0.09(0.3)$ & $63.61(64)$ & $>.05$ \\
\hline Trial number slope variance $-\operatorname{Var}\left(\mathrm{u}_{01}\right)$ & $0.00(0.07)$ & $77.37(64)$ & 0.12 \\
\hline Type of decision slope variance $-\operatorname{Var}\left(\mathrm{u}_{03}\right)$ & $0.27(0.52)$ & $81.17(63)$ & 0.06 \\
\hline
\end{tabular}

\section{Discussion}

The primary purpose of this study was to investigate the way people make choices under uncertainty. Because the decision-making process might vary according to the relevance of the situation, we expected that there would be differences in how people use information when faced with medical decisions vs. non-medical decisions.

The results supported our first hypothesis, which stated that people tend to choose the option that is superior on the common attribute more often when making medical choices, as compared to non-medical choices. One possible explanation is that when it comes to medical choices, people might use a strategy aimed at reducing postdecision regrets. Kivetz \& Simonson (2000) showed that when the participants in their study tried to interpret the unique attributes, and use them in the decision-making process, the choice was perceived as conflicting and difficult to make. Because decision conflict is associated with post-decision regret (Brehaut et al., 2003), people rely on common attributes as a strategy to minimize decision conflict, thus preventing regret. In support of this explanation, Charles, Ghafni, \&Whelan (1997) show that, even if they want information about the treatment options they have, patients do not necessarily want to be responsible for the treatment decisions, possibly in order to avoid the feeling of regret.

Connoly \& Zeelenberg (2002) reported that students who changed course section and found that they moved into a worse section than the one they left felt more regret compared to the students that had the same outcome imposed on them by another authority. Thus, it may be that people who decide for themselves expect to feel more regret if the outcome is bad than people who have the same outcome imposed on themselves from the outside.

Our second hypothesis stated that shorter response latencies predict people's tendency to choose the option that is superior on the common attribute. Results supported this idea, showing that a choice based on common attributes is predicted by less time spent on the decision, while more time spent on a decision predicts a choice that is based on the unique attributes. These findings are convergent with Kivetz and Simonson's (2000) conclusion that it is more demanding to take into account the unique attributes when choosing because this requires an additional effort: to make inferences about the missing values and include these inferences in the decision process, and this takes more time.

Future research should check whether the prescription of a timeframe for decision-making under uncertainty influences the type of strategy people use when making choices. Also, it would be interesting to see if imposing a timeframe (either less, or more than the average time people usually take) when making choices under uncertainty affects the self-reported perception of decision conflict and difficulty.

Our third hypothesis was that people's strategy to use common or unique attributes is linked to their tendency to endorse purity as a moral value. The data we collected showed that purity was not a significant predictor of how people used information in the decision-making process. Thus, this hypothesis did not receive empirical support. This finding seems divergent from previous studies, which stated a relationship between people's favorability towards moral purity, and vaccine hesitancy (Amin et al., 2017), or science skepticism (Rutjens, Sutton, \& van der Lee, 2018). One possible explanation for the divergence between the present results and those presented by Amin et al. (2017) or Rutjens, Sutton, \& van der Lee (2018) refers to the way the variables were operationalized. In the present study, we used a decision task that tapped participant's decisionmaking behavior, while Amin et al. (2017) or Rutjens, Sutton, \& van der Lee (2018) used self-report instruments to measure vaccine hesitancy, or the tendency to reject scientific information. Thus, it is possible that the results we obtained would not be statistically significant because purity was measured via self-report, while people's decision strategy was measured via a behavioral task. As Meyer et al. (2001) and Vazire (2006) underline, the relation between self- report measures and behavior tends to be modest. Using a bigger sample might help to detect the modest relationship between self- report measures and behavior, hence future studies could gather data from more participants, and employ a larger number of decision trials.

Last but not least, we found that gender is not a significant predictor of people's tendency to base their decision on common attributes, both for medical and nonmedical decisions. While the results did not support the direct hypothesis, we found that gender moderated the relationship between the type of decision and decision outcome. In the case of medical decisions, men tend to rely more on the known information, as compared to women, but this difference was no longer significant for nonmedical choices. A similar finding by Murtagh, \& Thomson (2006) is that women are more likely than men to prefer an active role when it comes to medical decisionmaking. As we did not approach this hypothesis, future studies should investigate the possible (negative) relation between preferring to play an active role in medical decision-making, and the tendency to rely on the known 
information when making a decision. Furthermore, future research could investigate potential gender differences regarding decision conflict, decision difficulty, and regret anticipation. Because women tend to rely on the unique attributes more than men do, when choosing between medical treatments, we would expect that women would also experience more decision conflict, decision difficulty and regret anticipation. Integrating the unique attributes of choices when making a decision requires additional inferences and effort, without the warranty of a good decision outcome. The awareness of uncertainty might, thus, lead to uncomfortable feelings of decision conflict and regret anticipation.

Limits

Since the study was conducted in a laboratory setting, the external validity of the results, and more specifically the ecological validity (i.e., the ability to extrapolate our findings to real-life decision making) is up for debate. Roe \& Just (2009) argue that researchers have to make a tradeoff between internal and external validity when choosing to conduct their investigations in the laboratory or naturalistic settings. We chose to focus on the internal validity of the study in order to clarify the variables involved in the decision-making process when facing incomplete information. Future research could focus on the external validity of the results, for instance by using verbal reports of the decision-making process in real-life medical and non-medical situations.

Next, it is possible that some attributes might have more weight than others. For instance, when it comes to one's health, the effectiveness of treatment might weigh more than its cost. In the present study, we did not control for this factor. Future research should include the type of information provided in the analysis, to verify if it accounts for variability in the decision-making process.

The small number of participants $(n=65)$ is a drawback of the present study, especially regarding the hypothesized effect of purity endorsement on medical decisions. Hence, in order to find an effect if there is one, a larger sample of participants would be needed to increase the study's statistical power.

Furthermore, Purity endorsement is measured via a self-report instrument while the medical scenarios requested a decision process that is similar to real life decision-processes. Ajzen (1987) shows that measures of traits or attitudes show only low correlations with specific actions.

Last but not least, research claims that there are individual differences in people's thinking style (Epstein, Pacini, Denes-Raj \& Heier, 1996). While some people tend to have a rational, deliberative approach, others take an intuitive or experiential outlook of the situations they encounter. The preference for intuition and/or the preference for deliberation might account for people's decision-making approach in situations with incomplete information. Thus, future research should also measure participants' thinking style in order to investigate if it accounts for any variability in decisions.

\section{Conclusion}

Though the structure of the information is kept identical, people approach medical decisions differently from non-medical, consumer decisions. While there is a general trend that people rather choose according to the common attributes of the alternatives, this tendency is significantly accentuated when they have to choose medical treatment. Also, people take less time to decide when they choose according to the common attributes of the alternatives.

Moral purity does not seem to be linked to medical decision-making, but this issue deserves further investigation, with bigger samples, other study designs (e.g., naturalistic studies), and different operationalization for the variables investigated (e.g., implicit measures of moral values). Finally, gender seems to moderate the way people make decisions, in the sense that women choose according to the unique attributes more often than men, but this difference only stands for medical choices; for nonmedical choices, there is no significant difference between men and women.

Taken together, the findings presented in this paper encourage future inquiries on the way people approach medical choices, both to clarify the way people use information in the decision-making process, and to identify how individual values guide people's judgment. Since today's patients are progressively encouraged to participate in decisions regarding their care, understanding their stand and judgment on the matter would bring valuable insights for physicians and policymakers alike.

\section{References}

Ajzen, I. (1987). Attitudes, traits, and actions: Dispositional prediction of behavior in personality and social psychology. Advances in Experimental Social Psychology, 1(20) 1-63. doi: 10.1016/S0065-2601(08)60411-6

Amin, A. B., Bednarczyk, R. A., Ray, C. E., Melchiori, K. J., Graham, J., Huntsinger, J. R., \& Omer, S. B. (2017). Association of moral values with vaccine hesitancy. Nature Human Behaviour, 1(12), 873. doi: 10.1038/s41562-017-0256-5

Brehaut, J. C., O'Connor, A. M., Wood, T. J., Hack, T. F., Siminoff, L., Gordon, E., \& Feldman-Stewart, D. (2003). Validation of a decision regret scale. Medical Decision Making, 23(4), 281-292. doi: 10.1177/0272989X03256005

Chalmers, I., \& Glasziou, P. (2009). Avoidable waste in the production and reporting of research evidence. The Lancet, $\quad 374(9683), \quad 86-89 . \quad$ doi: $\quad 10.1016 /$ S01406736(09)60329-9

Charles, C., Gafni, A., \& Whelan, T. (1997). Shared decisionmaking in the medical encounter: what does it mean? (or it takes at least two to tango). Social Science \& Medicine, 44(5), 681-692. doi: 10.1016/S0277-9536(96)00221-3

Connolly, T., \& Zeelenberg, M. (2002). Regret in decision making. Current Directions in Psychological Science, 11(6), 212-216 doi: 10.1111/1467-8721.00203

Croson, R., \& Gneezy, U. (2009). Gender differences in preferences. Journal of Economic Literature, 47(2), 44874. doi: 10.1257/jel.47.2.448

Dubé, E., Vivion, M., Sauvageau, C., Gagneur, A., Gagnon, R., \& Guay, M. (2016). "Nature does things well, why should we interfere?" Vaccine hesitancy among mothers. Qualitative Health Research, 26(3), 411-425. doi: 10.1177/1049732315573207

Epstein, S., Pacini, R., Denes-Raj, V., \& Heier, H. (1996). Individual differences in intuitive-experiential and analytical-rational thinking styles. Journal of Personality and Social Psychology, 71(2), 390. https://doi.org/10.1037/0022-3514.71.2.390

Frosch, D. L., \& Kaplan, R. M. (1999). Shared decision making in clinical medicine: past research and future directions. American Journal of Preventive Medicine, 17(4), 285-294. doi: 10.1016/S0749-3797(99)00097-5 
Galotti, K. M. (2005). Making decisions that matter: How people face important life choices. Mahwah, New Jersey: Lawrence Erlbaum Associates, Inc.

Gigerenzer, G. (2008). Why heuristics work. Perspectives on Psychological Science, 3(1), 20-29. doi: 10.1111/j.17456916.2008.00058.x

Gigerenzer, G., \& Todd, P. M. (1999). Fast and frugal heuristics: The adaptive toolbox. In Gigerenzer, G., Todd, P.M., \& the ABC Research Group (Ed.), Simple heuristics that make us smart (pp. 3-34). Oxford: Oxford University Press.

Graham, J., Nosek, B. A., Haidt, J., Iyer, R., Koleva, S., \& Ditto, P. H. (2011). Mapping the moral domain. Journal Of Personality And Social Psychology, 101(2), 366. doi: $10.1037 / \mathrm{a} 0021847$

Heitz, R. P. (2014). The speed-accuracy tradeoff: history, physiology, methodology, and behavior. Frontiers in Neuroscience, 8(8). doi: 10.3389/fnins.2014.00150

Kahn, B. E., \& Luce, M. F. (2003). Understanding high-stakes consumer decisions: mammography adherence following false-alarm test results. Marketing Science, 22(3), 393410. doi: $10.1287 / \mathrm{mksc} .22 .3 .393 .17737$

Keramati, M., Dezfouli, A., \& Piray, P. (2011). Speed/accuracy trade-off between the habitual and the goal-directed processes. PLoS Computational Biology, 7(5). doi: 10.5281/zenodo.45852

Kivetz, R., \& Simonson, I. (2000). The effects of incomplete information on consumer choice. Journal of Marketing Research, 37(4), 427-448. doi: 10.1509/jmkr.37.4.427.18796

Lipshitz, R., \& Strauss, O. (1997). Coping with uncertainty: A naturalistic decision-making analysis. Organizational Behavior and Human Decision Processes, 69(2), 149-163. doi: 10.1006/obhd.1997.2679

Meyer, G. J., Finn, S. E., Eyde, L. D., Kay, G. G., Moreland, K. L., Dies, R. R., et al. (2001). Psychological testing and psychological assessment: A review of evidence and issues. American Psychologist, 56(2), 128-165. doi: 10.1037/0003-066X.56.2.128

Politi, M. C., Han, P. K., \& Col, N. F. (2007). Communicating the uncertainty of harms and benefits of medical interventions. Medical Decision Making, 27(5), 681-695. doi: 10.1177/0272989X07307270

Rousell, G., \& Emenogu, B. (2009) HNM Workbook: An introduction to Hierarchical Nonlinear ModelingBernoulli Analysis. [PDF file]. Retrieved February 16, from

https://dugontario.files.wordpress.com/2013/12/bernoulliworkbook.pdf

Roe, B. E., \& Just, D. R. (2009). Internal and external validity in economics research: Tradeoffs between experiments, field experiments, natural experiments, and field data. American Journal of Agricultural Economics, 91(5), 1266 1271. doi: /10.1111/j.1467-8276.2009.01295.x

Rutjens, B. T., Sutton, R. M., \& van der Lee, R. (2018). Not all skepticism is equal: Exploring the ideological antecedents of science acceptance and rejection. Personality and Social Psychology Bulletin, 44(3), 384405. doi: 10.1177/0146167217741314

Say, R., Murtagh, M., \& Thomson, R. (2006). Patients' preference for involvement in medical decision making: a narrative review. Patient Education and Counseling, 60(2), 102-114. https://doi.org/10.1016/j.pec.2005.02.003

Simpson, A. (in press). Moral Foundations Theory: Background, Review, and Scaffolding for Future Research. In Encyclopedia of Personality and Individual Differences (pp. 1-19). Springer International Publishing

Slovic, P., \& MacPhillamy, D. (1974). Dimensional commensurability and cue utilization in comparative judgment. Organizational Behavior and Human Performance 11(2), 172-194. doi: 10.1016/00305073(74)90013-0

Tversky, A., \& Kahneman, D. (1992). Advances in prospect theory: Cumulative representation of uncertainty. Journal of Risk and Uncertainty, 5(4), 297-323. https://doi.org/10.1007/BF00122574

Vazire, S. (2006). Informant reports: A cheap, fast, easy method for personality assessment. Journal of Research in $\begin{array}{lr}\text { Personality, } & 40(1), \\ \text { https://10.1016/j.jrp.2005.03.003 }\end{array}$ 